\title{
Examining lifestyle behaviours and weight status of primary schoolchildren: using Mozambique to explore the data gaps in low- and middle-income countries
}

\author{
Taru Manyanga
}

\begin{abstract}
The emergency of malnutrition and physical inactivity among children as serious public health challenges in low- and middle-income countries (LMICs) is concerning and requires urgent attention. The main objective of this dissertation was to examine relationships between lifestyle behaviours and weight status among schoolchildren in Mozambique and use findings to highlight important data gaps that exist in LMICs. Narrative literature searches conducted identified data gaps and research needs. A published protocol was used for this dissertation $(n=683)$ to facilitate data comparability. Anthropometric and accelerometry data were objectively measured while data about lifestyle behaviours and environmental factors were collected using context-adapted questionnaires. As part of this dissertation, 6 manuscripts were developed and submitted for publication in peer-reviewed scientific journals. Results showed a dearth of information and that overweight/obesity is an emerging public health concern, especially among urban children. Moderate- to vigorous-intensity physical activity (MVPA), active transport, and maternal body mass index (BMI) were important modifiable correlates of weight status for Mozambican children. Distinct differences in prevalences of lifestyle behaviours were observed between urban and rural children in Mozambique. Compared with children from 12 other countries, children from Mozambique had lower BMI, higher daily MVPA, lower daily sedentary time, and comparable sleep duration. Linear distributions of study site-specific BMI, minutes of daily MVPA, and daily sedentary time by country human development index were observed. Findings revealed important differences between urban and rural children, supporting the need to include both in study samples and especially in LMICs where most people live in rural areas.
\end{abstract}

Received 17 November 2019. Accepted 27 November 2019.

T. Manyanga.*,† Healthy Active Living and Obesity Research Group, Children’s Hospital of Eastern Ontario Research Institute, Ottawa, ON K1H 8L1, Canada.

Email for correspondence: tmanyanga@cheo.on.ca.

*Advisor: Mark S. Tremblay, Healthy Active Living and Obesity Research Group, Children’s Hospital of Eastern Ontario Research Institute, Ottawa, ON K1H 8L1, Canada.

†Taru thanks Dr. Tremblay and the other co-authors on the 6 manuscripts in his dissertation.

Copyright remains with the author(s) or their institution(s). Permission for reuse (free in most cases) can be obtained from RightsLink. 